\title{
Microfluidics for antibody-independent separation of circulating tumor cells
}

\author{
Vijay Duryodhan ${ }^{1}$ and Shiv Govind Singh ${ }^{1}$ * \\ ${ }^{1}$ Department of Electrical Engineering, IIT Hyderabad
}

\begin{abstract}
Separation of circulating tumor cells (CTC) from the blood without using antibodies is a key challenge in the diagnosis of cancer. This article has reviewed few antibody-independent techniques governed by cell size on microfluidics platform. Microdevices employing micro-pore filters, dielectrophoresis, acoustophoresis and inertia that is popular in various research labs are studied in detail from the literature and discussed shortly. The inertia based technique in spiral microchannel seems to be more promising towards the development of future point-of-care (POC) microdevices for CTCs separation.
\end{abstract}

Keywords: antibody-independent separation; circulating tumor cells; microfluidics; microdevices

*Corresponding author: Dr. Shiv Govind Singh, Department of Electrical Engineering, Indian Institute of Technology Hyderabad, Sangareddy, Khandi, Telangana 502285. Email: sgsingh@iith. ac.in

Received 20 January 2017; Revised 17 March 2017; Accepted 24 March 2017; Published 30 March 2017

Citation: Duryodhan V, Singh SG. Microfluidics for antibodyindependent separation of circulating tumor cells. J Med Sci Res. 2017; 5(2):61-65. DOI: http://dx.doi.org/10.17727/ JMSR.2017/5-12

Copyright: (C) 2017 Duryodhan V et al. Published by KIMS Foundation and Research Center. This is an open-access article distributed under the terms of the Creative Commons Attribution License, which permits unrestricted use, distribution, and reproduction in any medium, provided the original author and source are credited.

\section{Introduction}

Microfluidics deals with the manipulation of micron litre of fluid volume inside the micron sized flow conduits. Microfluidics based devices provide various advantages such as the requirement of less samples/ reagents volume, fast reaction and therefore quick response time (because of large surface-to-volume ratio), laminar flow, low cost, high throughput, minimal sample loss (because of one step process). Because of these advantages microfluidics based devices find applications in various industries such as, electronics, chemical, medical, agriculture, marine etc. The global market for microfluidics based devices is expected to reach $\$ 3.57$ billion and $\$ 8.64$ billion respectively by 2018 and 2023 [1, 2] from its earlier value of $\$ 1.59$ billion in 2013. Such economic prediction is usually based on the market requirement and the industries investment towards research and development. However, despite of such promising economic survey very few micro devices are successfully able to launch in the consumer market. Difficulty lies in terms of dependency 
and complex integration of the microdevices with conventional scale instrumentation.

This review article discusses the progress of one such microdevice needed in diagnosis of cancer. After heart diseases cancer is the second leading cause of morbidity and mortality worldwide. Approximately 14 million new cases are reported in 2012 which is expected to rise by about $70 \%$ leading to 23.8 million in the next two decades [3]. According to the World Health Organization (WHO), at least 30\% of these deaths would be preventable, if patients are diagnosed and treated before cancer metastases occur which is believed to be a major cause of cancer related deaths. Cancer metastases arise after distribution of CTCs into the blood stream from its primary or secondary tumor sites. Identification of CTCs in the blood stream is non-trivial given the frequency of occurrence i.e. $\sim 1$ in $10^{7}$ leukocytes. Therefore, separation of CTCs from the population of healthy blood cells is foremost step in the study of cancer cells. Ideal separation technique must be able to provide high purity and recovery rates while maintaining CTCs alive and intact for morphological and molecular analysis. High throughput is the other requirement to achieve more alive CTCs in a given time span. Numerous approaches developed, to separate CTCs at the conventional scale lack the requirements mentioned above. The only FDA approved technique by CellSearch ${ }^{\mathrm{TM}}$ is well-known for batch processing [4]. However, this system employs an immuno-magnetic approach to capture CTCs which leads to loss of rare cell (CTCs) during their tagging with magnetic beads using multiple pipetting. Also, this technique requires antibodies and valid only for isolating known cancer cells. Microfluidics based cell separation is therefore useful to overcome the limitations in the existing conventional scale system. This review article summarizes some of the promising microfluidics based cell separation techniques available in the literatures.

\section{Microfluidics based cell/CTCs separation techniques}

Microfluidic based CTCs separation technique can be classified into passive and active methods. It is also classified into, with and without biomarker based approach. Biomarker based approaches are more specific but only applicable to the known cancer cells. Further, the cost of antibodies makes this approach unsustainable. Therefore, this work mainly focuses on the methods which are independent of biomarkers.

\section{Filter based method}

Tan et al. proposed the label-free approach for separating cancer cells from breast and colon origin from the blood using crescent-shaped filter, microdevice casted into poly(dimethylsiloxane) (PDMS) and bonded on glass substrate [5]. They utilized the larger size and stiffer characteristic of the cancer cells compared to blood constituents for this purpose. Isolation efficiency of the proposed microdevice is reported to be $80 \%$ for separation of MCF-7, MDA-MB231 and HT-29.

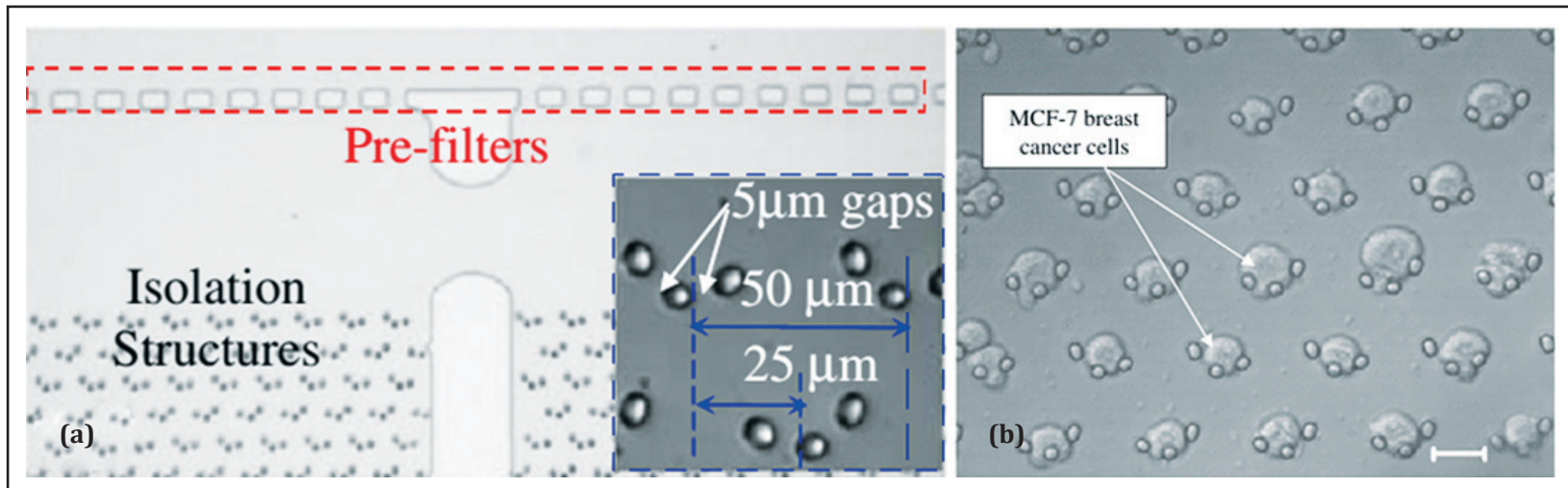

Figure 1: (a) Crescent-shaped filter and (b) Captured MCF-7 breast cancer cells [5].

Zheng et al. reported a three dimensional microfilter device where position of the top and bottom membrane shifted as shown in Figure 2 [6]. Bottom filter is etched in silicon wafer using wet etching whereas, top filter patterned in parylene using oxygen plasma. A known number of CFSE labeled 
MCF-7 cells were introduced into the healthy blood. They reported $86.5 \pm 5.3 \%$ capture efficiency which is comparable to their earlier reported efficiency for two dimensional filter based microdevice. However, careful comparison of 3D and 2D filter shows that 3D filter is able to capture intact MCF-7 cells preserving their membrane whereas 2D filter fails to do so and cells undergo lysis. Micropore filtration based microdevices facilitate the label-free approach to enrich the CTCs using the biophysical rather biochemical characteristics of the cancer cells. However, these devices are prone to clogging and therefore not so popular among research communities.
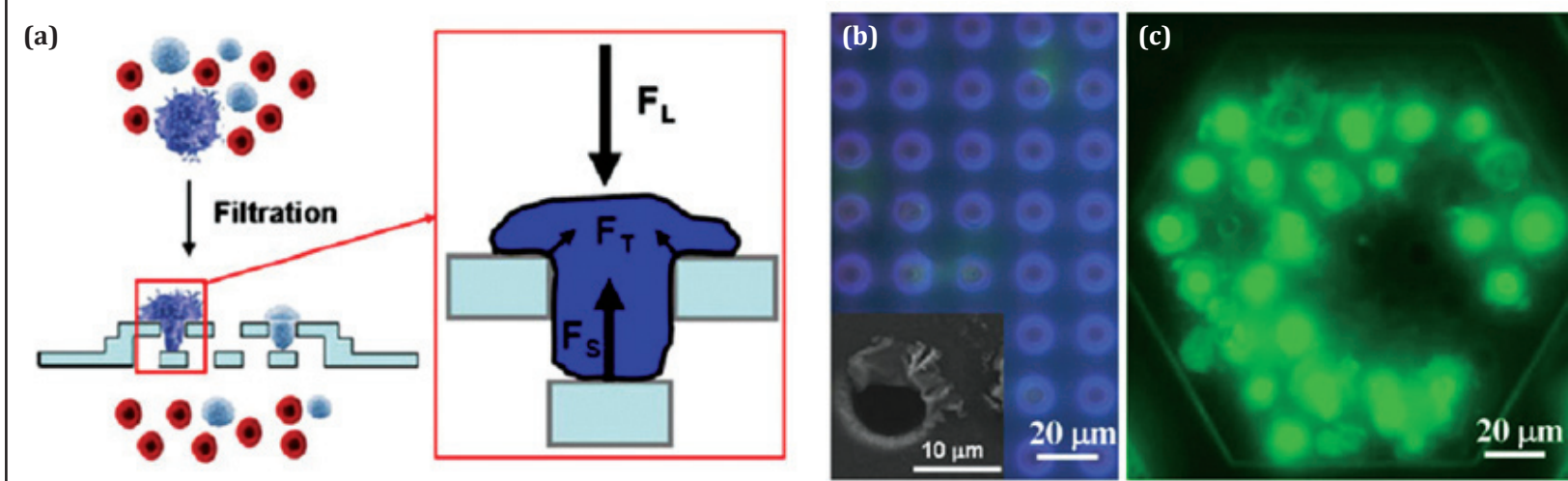

Figure 2: (a) Schematic diagram showing concept of 3D filter microdevice; (b) 2D filter with cell lysis, ruptured cell membrane deposited at the edge of micropores; (c) 3D filter with captured intact cells as shown by bright fluoresce spots [6].

\section{Dielectrophoresis based method}

Dielectrophoresis (DEP) based cell separation is an antigen-independent phenomenon that depends on cell dielectric properties which in turn function of cell morphology and membrane surface area. Few research groups explored the possibility of using DEP to separate the CTCs. Shim et al. developed the continuous flow dielectrophoretic fieldflow-fractionation (DEP-FFF) approach in which continuous flow of a low conductivity eluate is maintained to have Poisseuille flow [7] (Figure 3a). This method is reported to be capable of separating the CTCs of any type irrespective of their tissue type and origin. This process is also capable of processing large sample volume $(10 \mathrm{ml})$ compared to only DEP based system. DEP based micro devices provide good capturing efficiency of around $90 \%$.
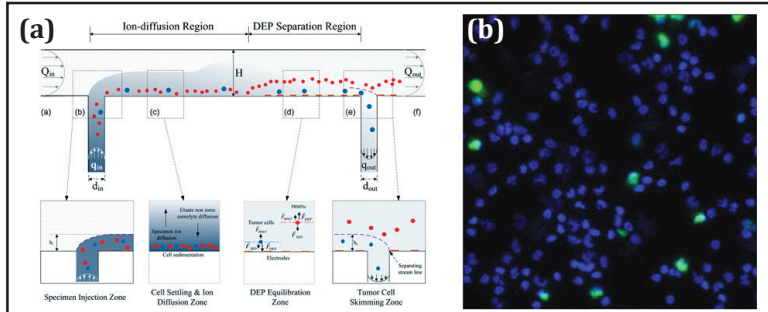

Figure 3: (a) Schematic diagram showing concept of DEPFFF; (b) Collected CTCs from the blood sample of a patient with colon cancer, green fluorescence indicates the staining of cytokeratin in the tumor cells by FITC-conjugate CK3-6H5 antibodies [7].

\section{Acoustophoresis based method}

Acoustophoresis is the method of separating particles using acoustic radiation resonance. Flowing particles of different size, shape first align at the nodal points of the interfering waves as shown at section a-a (Figure 4). These aligned particles are

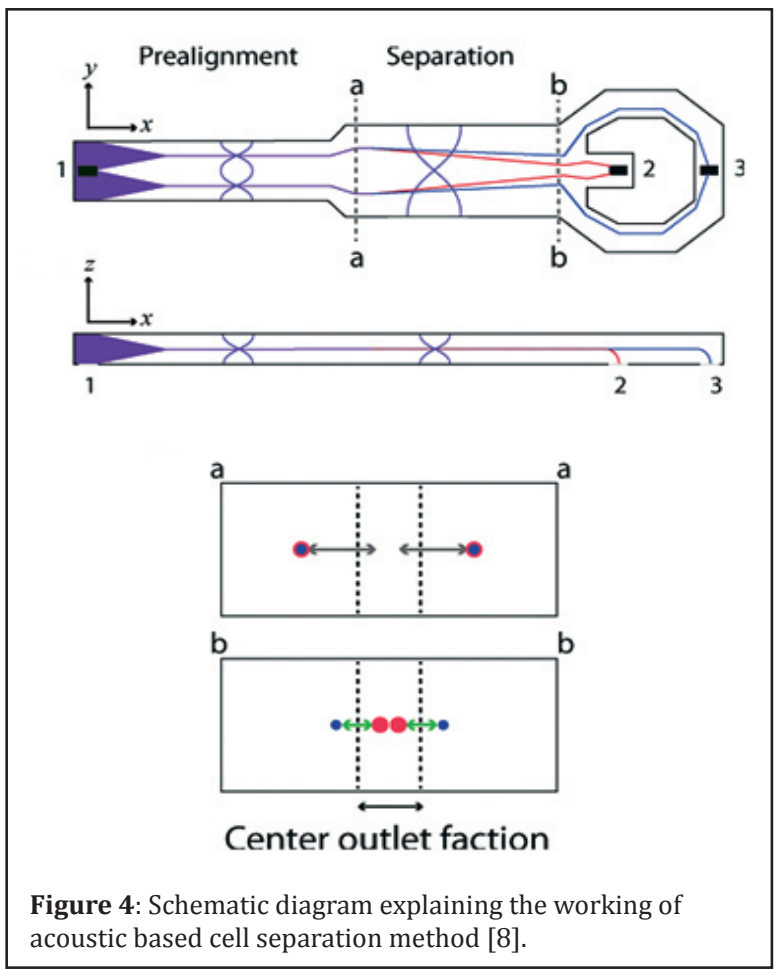


further separated out with larger particle flows at the center and smaller particle away from the center as shown at section b-b (Figure 4a, c). Antfolk et al. design micro-chip, based on acoustophoresis and fabricated in silicon using wet etching process [8]. Their experiments with Prostate cancer cells spiked in WBC enriched blood exhibits $86.5 \pm 6.7 \%$ recovery of the cancer cells which subsequently improves to $94.8 \pm 2.8 \%$ with the increase in acoustic intensity but with more contamination with WBC.
Hur et al. employed hydrodynamic flow separation technique in microchannel with sudden expansioncontraction using size of the cell as a biomarker [9] (Figure 5). The approach utilizes irreversible migration of particles into microscale vortices. The microfluidic device is fabricated in PDMS using soft lithography. The capturing efficiency for breast cancer cells (MCF-7) and cervical cancer cells (HeLa) are reported respectively $23 \%$ and $10 \%$; which is substantially less compared to the filter based techniques discussed above.

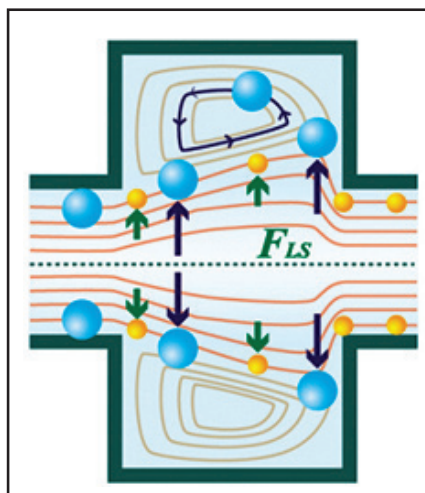

(a)

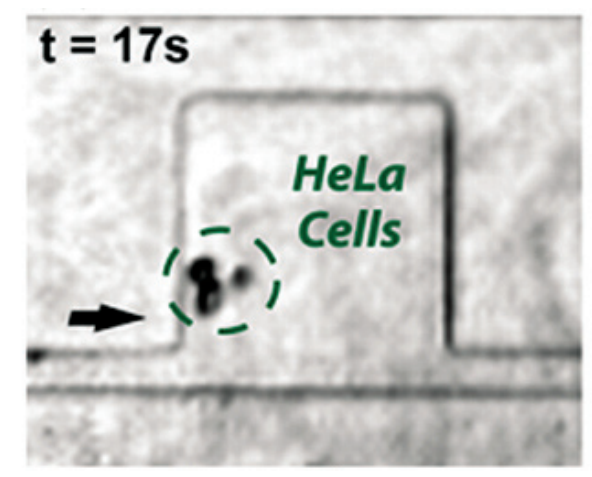

(b)

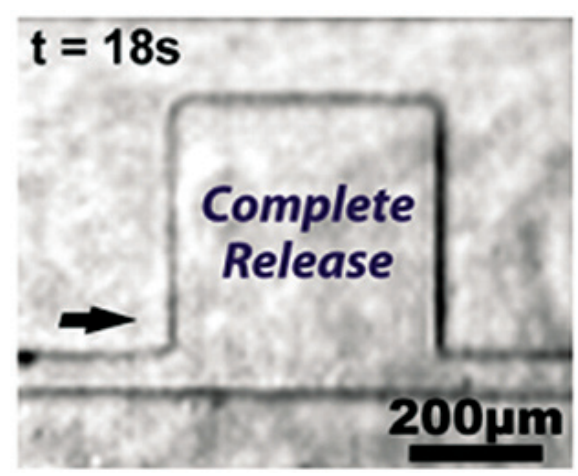

(c)

Figure 5: Inertia based method (a) Schematic representation of the concept of size based cell capturing in laminar microscale vortices; capture (b) and release (c) of cervical cancer cell (HeLa) from the cavity [9].

Based on the observation by Segre and Silberberg [10], it is known that particles migrate laterally and attain the equilibrium position inside the flow conduits based on their sizes. Accordingly, smaller particle migrates towards the wall while larger stabilizes near to the center of microchannel. This phenomenon is affected by the ratio of particleto-channel diameter which is further validated by the microfluidic community. Extensive research is carried out to understand this lateral migration. Recently, research community started exploring the possibility of using lateral migration of particles along with the dean vortices to separate the rare biological cells for disease diagnosis. For an illustration, Warkiani et al. proposed another labelfree approach for continuous separation of cancer cells from the whole blood [4]. This is the size based hydrodynamically particle separation method employing dean vortices. Spiral microchannel with appropriate dimensions and having two full turns fabricated in PDMS using soft lithography. Blood with RBC lysis and CTC pass through the microdevice in which CTCs are separated at the inner wall and rest flush out from the outer wall of the spiral microchannel (Figure 6). This device reported to separate $\geq 85 \%$ of the cancer cells.

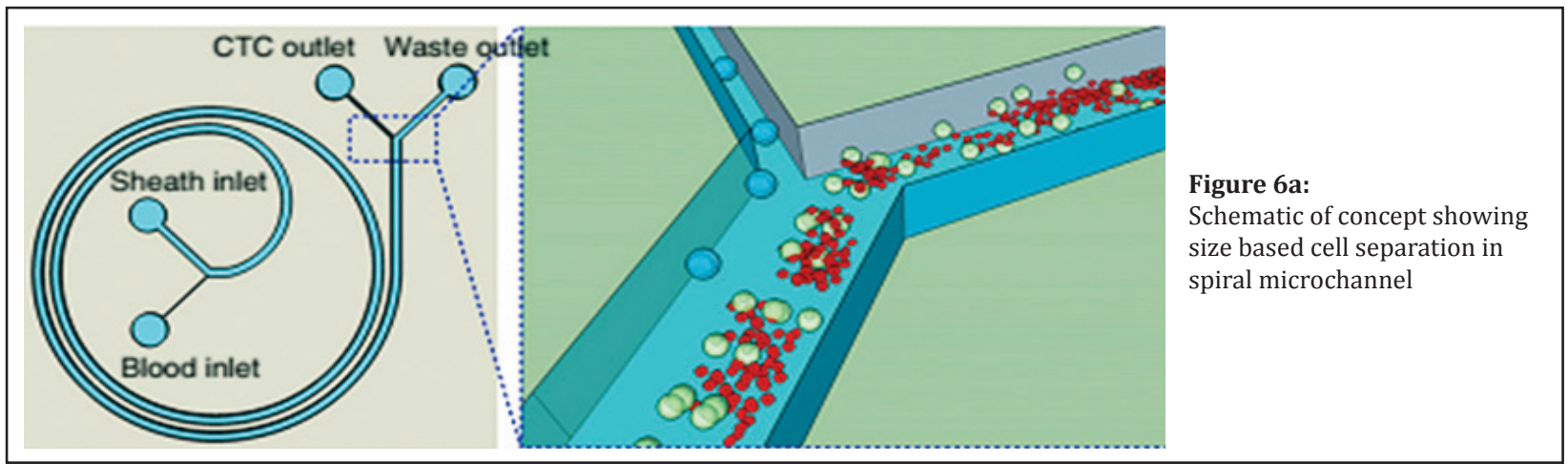




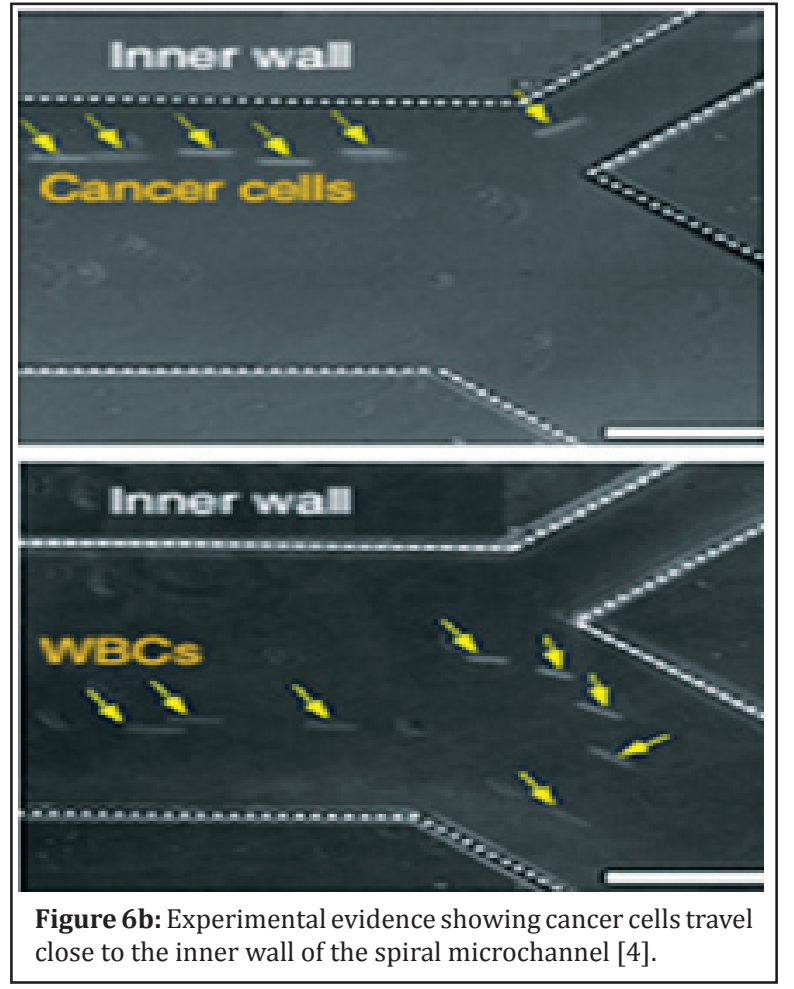

\section{Conclusions}

The rise in cancer related diseases worldwide forced the biomedical industries to explore the unconventional diagnostic methods. Separation of unknown circulating tumor cells from the blood without use of antibodies is foremost challenge in this direction. However, evolution in the field of microfluidics brings out various promising techniques to separate CTCs in last decade. This article reviewed some of the techniques that are antibody-independent and governed by size, shape, deformability of the CTCs. In this direction filter, electrophoresis, acoustophoresis and inertia based methods are discussed. Filter based microdevices are simple passive device but prone to the issue of clogging and therefore not suitable for continuous separation application. Electrophoresis and acoustophoresisareactivetechniquesintegrated with complex external circuits to generate respectively electric and acoustic fields. Spiral microchannel based technique is a very simple passive technique solely governed by the size of CTCs. This can be used in parallel to increase the throughput and in series to enhance the purity. Based on the literatures it seems that spiral microchannel based designs are more promising in developing future lab-on-chip or optimistically point-of-care (POC) microdevice.

\section{Conflicts of interest}

Authors declare no conflicts of interest.

\section{References}

[1] Microfluidics market by materials (polymers, silicon, glass), pharmaceuticals (microreactors, toxicity screening, lab on chip, proteomic \& genomic analysis) drug delivery devices (microneedles, micropumps), IVD (POC) - Global Trends \& Forecast to 2018. Markets and Markets. 2013.

[2] Microfluidic Technologies: Biopharmaceutical and Healthcare Applications 2013-2023. Visiongain. 2013.

[3] Media center, Cancer fact sheet. World Health Organization (WHO). 2017.

[4] Warkiani ME, Khoo BL, Wu L, Tay AK, Bhagat AA, et al. Ultrafast, label-free isolation of circulating tumor cells from blood using spiral microfluidics. Nat Protoc. 2016; 11(1):134148.

[5] Tan SJ, Yobas L, Lee GY, Ong C N, Lim CT. Microdevice for the isolation and enumeration of cancer cells from blood. Biomed Microdevices. 2009; 11(4):883-892.

[6] Zheng S, Lin HK, Lu B, Williams A, Datar R, et al. 3D microfilter device for viable circulating tumor cell (CTC) enrichment from blood. Biomed Microdevices. 2011; 13(1):203-213.

[7] Shim S, Stemke-Hale K, Tsimberidou AM, Noshari J, Anderson TE, et al. Antibody-independentisolation of circulating tumor cells by continuous-flow dielectrophoresis. Biomicrofluidics. 2013; 7(1):011807.

[8] Antfolk M, Antfolk C, Lilja H, Laurell T, Augustsson P. A single inlet two-stage acoustophoresis chip enabling tumor cell enrichment from white blood cells. Lab Chip. 2015; 15(9):2102-2109.

[9] Hur SC, Mach AJ, Carlo DD. High-throughput size-based rare cell enrichment using microscale vortices. Biomicrofluidics. 2011; 5(2):022206.

[10] Segre G, Silberberg A. Radial particle displacements in poiseuille flow of suspensions. Nature.1961; 189:209-210, 1961. 